University of Nebraska - Lincoln

DigitalCommons@University of Nebraska - Lincoln

Faculty Publications from the Department of Engineering Mechanics

2009

\title{
Optimal electrode shape and size for shear mode thin film acoustic wave resonators
}

L. M. Xu

University of Electronic Science and Technology, Chengdu, China

B. B. Tang

Huazhong University of Science and Technology, Wuhan, China

Y. T. Hu

Huazhong University of Science and Technology, Wuhan, China

H. Fan

Nanyang Technological University, Singapore

J. S. Yang

University of Nebraska-Lincoln, jyang1@unl.edu

Follow this and additional works at: https://digitalcommons.unl.edu/engineeringmechanicsfacpub

Part of the Mechanical Engineering Commons

Xu, L. M.; Tang, B. B.; Hu, Y. T.; Fan, H.; and Yang, J. S., "Optimal electrode shape and size for shear mode thin film acoustic wave resonators" (2009). Faculty Publications from the Department of Engineering Mechanics. 74.

https://digitalcommons.unl.edu/engineeringmechanicsfacpub/74

This Article is brought to you for free and open access by the Mechanical \& Materials Engineering, Department of at DigitalCommons@University of Nebraska - Lincoln. It has been accepted for inclusion in Faculty Publications from the Department of Engineering Mechanics by an authorized administrator of DigitalCommons@University of Nebraska - Lincoln. 


\title{
Optimal electrode shape and size for shear mode thin film acoustic wave resonators
}

\author{
L. M. Xu, ${ }^{1}$ B. B. Tang, ${ }^{2}$ Y. T. Hu, ${ }^{2}$ H. Fan, ${ }^{3}$ and J. S. Yang ${ }^{4, a)}$ \\ ${ }^{1}$ Institute of Astronautics and Aeronautics, University of Electronic Science and Technology, \\ Chengdu 610054, People's Republic of China \\ ${ }^{2}$ School of Civil Engineering and Mechanics, Huazhong University of Science and Technology, Wuhan, \\ Hubei 430074, People's Republic of China \\ ${ }^{3}$ School of Mechanical and Aerospace Engineering, Nanyang Technological University, \\ Singapore 639798, Singapore \\ ${ }^{4}$ Department of Engineering Mechanics, University of Nebraska, Lincoln, Nebraska 68588-0526, USA
}

(Received 22 September 2009; accepted 16 November 2009; published online 7 December 2009)

\begin{abstract}
We study electrode shape and size for plates of hexagonal crystals or polarized ceramics with the sixfold axis or the poling direction parallel to the plate surfaces so that they can be excited into thickness-shear vibration by an electric field in the plate thickness direction. Electrode size and shape optimal in the sense that they satisfy the criterion of Bechmann's number in every direction are determined. () 2009 American Institute of Physics. [doi:10.1063/1.3271981]
\end{abstract}

Electrodes are necessary parts of piezoelectric resonators and other devices. They are needed for electrically exciting mechanical vibrations. Impedance of resonant piezoelectric devices is an important design consideration and is calculated from the charge (or current) and voltage on the electrodes. In plate piezoelectric resonators, electrode mass is known to be responsible for energy trapping of thicknessshear (TSh) modes with which the vibration is confined within or close to the electroded region. Energy trapping is crucial to device mounting and frequency stability. Recently, due to the need of device miniaturization, there has been growing research interest in the effect of electrode configuration. Electrodes of varying thickness have been shown effective in producing strong energy trapping. ${ }^{1-5}$ Nonuniform electrodes provide an alternative to contoured resonators with varying thickness which have been used for strong energy trapping for a long time and are known to be difficult to make. Electrode shape also needs to be optimized. At present, circular and rectangular electrodes are routinely used in manufacturing. In Ref. 6, it was recently shown that electrodes with corners cause field concentration and should be avoided in general.

An important theoretical result on electrode shape and size was given by Mindlin, ${ }^{7}$ who obtained a formula for the optimal electrode shape and size of singly rotated AT-cut quartz plate resonators using two-dimensional plate equations. The optimal electrodes determined in Ref. 7 satisfy the criterion of Bechmann's number ${ }^{7}$ in every direction so that when the resonator is in TSh vibration the entire electroded region vibrate in phase. When the electrodes are larger than the optimal ones, the vibration modes have nodal lines under the electrodes which cause charge cancellation on the electrodes. When the electrodes are smaller than the optimal ones, the vibration modes are not fully made use of in terms of collecting charges for larger capacitance. Numerical results based on the formula in Ref. 7 were presented in Ref. 8 . The optimal electrodes determined by Mindlin for AT-cut quartz plates are nearly elliptical, which shows that the com-



monly used rectangular and circular electrodes deviate significantly from the optimal electrodes, and therefore significant improvement in resonator performance can be expected using the optimal electrodes. The result in Refs. 7 and 8 were recently generalized to the case of doubly rotated stress compensated (SC)-cut quartz plate resonators. ${ }^{9}$ The formulas and results in Refs. 7 through 9 are valid for quartz only.

Piezoelectric crystals of $6 \mathrm{~mm}$ symmetry including $\mathrm{ZnO}$ and AlN are of current research interest for thin-film resonators. Thin films of $6 \mathrm{~mm}$ crystals with normal, in-plane, and tilted sixfold axes can all be produced. When the sixfold axis is parallel to the plate surfaces (in-plane sixfold axis), the plate can be excited into TSh motion by an electric field in the plate thickness direction. In this letter, we determine optimal electrodes for plates of $6 \mathrm{~mm}$ crystals with in-plane sixfold axis in TSh vibration. A simple formula that determines the size and shape of the optimal electrodes is obtained. Numerical and graphical results of common $6 \mathrm{~mm}$ crystals are presented. Polarized ceramics are also common piezoelectric materials. Their material tensors have the same structures as those of $6 \mathrm{~mm}$ crystals. Therefore, our analysis is also valid for polarized ceramics with in-plane poling, which is a common device configuration.

Consider an unbounded, partially electroded plate of $6 \mathrm{~mm}$ crystals with in-plane sixfold axis as shown in Fig. 1. The direction of the sixfold axis is indicated by P. The plate has a thickness $2 h$ and a mass density $\rho$. The electrodes are with thickness $2 h^{\prime}$ and mass density $\rho^{\prime}$. The electrodes are

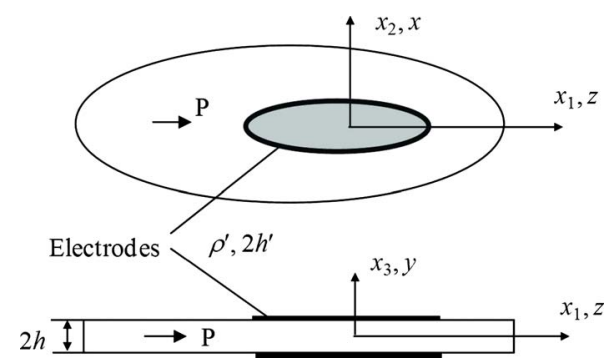

FIG. 1. (Color online) A partially electroded piezoelectric plate and coordinate system. 
assumed to be thin. Only their inertia will be considered. Their stiffness will be neglected. ${ }^{7}$ For the purpose of this letter, we only need to consider free vibrations with shorted electrodes. Two coordinate systems are used. $(x, y, z)$ is for defining the material constants such that the sixfold axis is along $z$. $\left(x_{1}, x_{2}, x_{3}\right)$ is for the plate equations ${ }^{10}$ to be used in which the plate normal is $x_{3}$. We summarize the plate equations below. Under a thick electric field, the plate in Fig. 1 is excited into TSh vibration with the following approximate displacement and electric potential fields:

$$
\begin{aligned}
& u_{1}\left(x_{1}, x_{2}, x_{3}, t\right) \cong x_{3} u_{1}^{(1)}\left(x_{1}, x_{2}, t\right), \quad u_{2} \cong 0, \quad u_{3} \cong 0, \\
& \phi\left(x_{1}, x_{2}, x_{3}, t\right) \cong x_{3} \phi^{(1)}\left(x_{1}, x_{2}, t\right) .
\end{aligned}
$$

The equations governing $u_{1}^{(1)}$ and $\phi^{(1)}$ were given in Ref. 10 which, when specialized to $6 \mathrm{~mm}$ crystals, are

$$
\begin{aligned}
& \gamma_{11} u_{1,11}^{(1)}+c_{44} u_{1,22}^{(1)}+\psi_{11} \phi_{, 11}^{(1)}+e_{15} \phi_{, 22}^{(1)}-3 h^{-2}\left(\kappa_{1}^{2} c_{44} u_{1}^{(1)}\right. \\
& \left.\quad+\kappa_{1} e_{15} \phi^{(1)}\right)=\rho \ddot{u}_{1}^{(1)}, \\
& \psi_{11} u_{1,11}^{(1)}+e_{15} u_{1,22}^{(1)}-\zeta_{11} \phi_{, 11}^{(1)}-\varepsilon_{11} \phi_{, 22}^{(1)}-3 h^{-2}\left(\kappa_{1} e_{15} u_{1}^{(1)}\right. \\
& \left.\quad-\varepsilon_{11} \phi^{(1)}\right)=0,
\end{aligned}
$$

where

$$
\begin{aligned}
& \gamma_{11}=c_{33}^{E}-\left(c_{13}^{E}\right)^{2} / c_{11}^{E}, \quad c_{44}=c_{44}^{E}, \\
& \psi_{11}=e_{33}-e_{31} c_{13}^{E} / c_{11}^{E}, \\
& \zeta_{11}=\varepsilon_{33}^{S}+e_{31}^{2} / c_{11}^{E}, \quad \varepsilon_{11}=\varepsilon_{11}^{S} .
\end{aligned}
$$

In order to determine the optimal electrodes, we need to analyze the unelectroded and the electroded portions of the plate separately. First we consider unelectroded plates. For an unelectroded plate the shear correction factor $\kappa_{1}^{2}$ is

$$
\kappa_{1}^{2}=\frac{\pi^{2}}{12} .
$$

For determining the optimal electrodes, we need the dispersion relation for TSh waves governed by Eqs. (2) and (3). For waves propagating in a direction described by $\psi$ with a wavelength $\lambda$, we let

$$
\begin{aligned}
& u_{1}^{(1)}=A \exp \left[\mathrm{i} \frac{2 \pi}{\lambda}\left(x_{1} \cos \psi+x_{2} \sin \psi\right)\right] \exp (-\mathrm{i} \omega t), \\
& \phi^{(1)}=B \exp \left[\mathrm{i} \frac{2 \pi}{\lambda}\left(x_{1} \cos \psi+x_{2} \sin \psi\right)\right] \exp (-\mathrm{i} \omega t),
\end{aligned}
$$

where $A$ and $B$ are undetermined constants. Substitution of Eq. (6) into Eqs. (2) and (3) yields two linear, homogeneous equations for $A$ and $B$. For nontrivial solutions, the determinant of the coefficient matrix of the equations must vanish, which gives the frequency equation that determines the following dispersion relation:

$$
\begin{aligned}
\rho \omega^{2}= & \gamma_{11}\left(\frac{2 \pi}{\lambda} \cos \psi\right)^{2}+c_{44}\left(\frac{2 \pi}{\lambda} \sin \psi\right)^{2}+3 h^{-2} \kappa_{1}^{2} c_{44} \\
& +\frac{\left[\psi_{11}\left(\frac{2 \pi}{\lambda} \cos \psi\right)^{2}+e_{15}\left(\frac{2 \pi}{\lambda} \sin \psi\right)^{2}+3 h^{-2} \kappa_{1} e_{15}\right]^{2}}{\zeta_{11}\left(\frac{2 \pi}{\lambda} \cos \psi\right)^{2}+\varepsilon_{11}\left(\frac{2 \pi}{\lambda} \sin \psi\right)^{2}+3 h^{-2} \varepsilon_{11}} .
\end{aligned}
$$

In particular, the cutoff frequency with $\lambda=\infty$ is given by

$$
\omega^{2}=\frac{\pi^{2} \bar{c}_{44}}{4 \rho h^{2}}
$$

where

$$
\bar{c}_{44}=c_{44}\left(1+k_{15}^{2}\right), \quad k_{15}^{2}=\frac{e_{15}^{2}}{\varepsilon_{11} c_{44}} .
$$

Next we consider electroded plates. For an electroded plate with shorted electrodes, $\phi^{(1)}=0$ and no equation for $\phi^{(1)}$ is needed. When the electrode mass inertia is considered, it affects the governing equation of $u_{1}^{(1)}$ through a mass ratio term $R .^{11}$ The governing equation for $u_{1}^{(1)}$ becomes

$$
\gamma_{11} u_{1,11}^{(1)}+c_{44} u_{1,22}^{(1)}-3 h^{-2} \bar{\kappa}_{1}^{2} c_{44} u_{1}^{(1)}=\rho(1+3 R) \ddot{u}_{1}^{(1)},
$$

where the electrode/plate mass ratio $R$ is ${ }^{12}$

$$
R=\frac{2 \rho^{\prime} h^{\prime}}{\rho h} .
$$

For an electroded plate, in the manner of Ref. 12, by requiring the cutoff frequency of the fundamental TSh mode from the three-dimensional equations and the plate equations to be equal, the shear correction factor $\bar{\kappa}_{1}^{2}$ can be determined as

$$
\begin{aligned}
& \bar{\kappa}_{1}^{2}=\frac{\pi^{2}}{12}\left(1+R-\frac{8}{\pi^{2}} \bar{k}_{15}^{2}\right) \frac{\bar{c}_{44}}{c_{44}}, \\
& \bar{k}_{15}^{2}=\frac{e_{15}^{2}}{\varepsilon_{11} \bar{c}_{44}} .
\end{aligned}
$$

Let

$$
u_{1}^{(1)}=C \exp \left[\mathrm{i} \frac{2 \pi}{\lambda}\left(x_{1} \cos \psi+x_{2} \sin \psi\right)\right] \exp (-\mathrm{i} \omega t) .
$$

where $C$ is an undetermined constant. Substitution of Eq. (13) into Eq. (10) gives

$$
\begin{aligned}
\rho(1+3 R) \omega^{2}= & \gamma_{11}\left(\frac{2 \pi}{\lambda} \cos \psi\right)^{2}+c_{44}\left(\frac{2 \pi}{\lambda} \sin \psi\right)^{2} \\
& +3 h^{-2} \bar{\kappa}_{1}^{2} c_{44} .
\end{aligned}
$$

In the spirit of Ref. 7, the optimal dimension $L$ of the electrode in the direction $\psi$ is the wavelength of the TSh wave in that direction in an electroded plate at the cutoff frequency of the same wave in an unelectroded plate. Hence, from Eqs. (8) and (14), $L$ is determined by the following equation as a function of $\psi$ : 


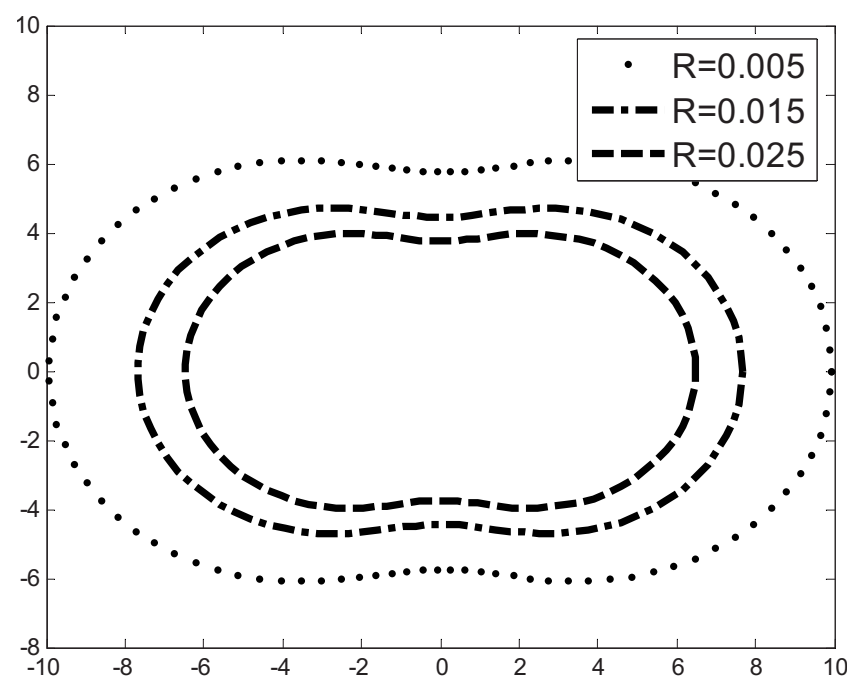

FIG. 2. Optimal electrodes for AlN plates.

$$
\begin{aligned}
\rho(1+3 R) \frac{\pi^{2} \bar{c}_{44}}{4 \rho h^{2}}= & \gamma_{11}\left(\frac{2 \pi}{L} \cos \psi\right)^{2}+c_{44}\left(\frac{2 \pi}{L} \sin \psi\right)^{2} \\
& +3 h^{-2} \bar{\kappa}_{1}^{2} c_{44} .
\end{aligned}
$$

An explicit expression of the normalized $L$ can be obtained as

$$
l=\frac{L}{2 h}=\left[\frac{\gamma_{11}(2 \pi \cos \psi)^{2}+c_{44}(2 \pi \sin \psi)^{2}}{(1+3 R) \pi^{2} \bar{c}_{44}-12 \bar{\kappa}_{1}^{2} c_{44}}\right]^{1 / 2} .
$$

For numerical examples we consider $\mathrm{ZnO}$ and AlN. The material constants can be found in Ref. 13. The resonator thickness is fixed to be $2 h=1 \mathrm{~mm}$. We calculate $r=l / 2$ versus $\psi$ and plot $l$ in the direction of $\psi$ for different values of $R$. The results are shown in Figs. 2 and 3 for AlN and $\mathrm{ZnO}$, respectively. The figures show that the optimal electrodes deviate considerably from common circular or rectangular shapes. Therefore, significant improvement of device performance can be expected when optimal electrodes are used. The figures also show that for larger values of $R$, the optimal electrodes become smaller. This is consistent with our previous knowledge on energy trapping ${ }^{1-5}$ and is as expected.

In summary, optimal electrode shape and size are determined for plates of crystals of $6 \mathrm{~mm}$ symmetry with in-plane

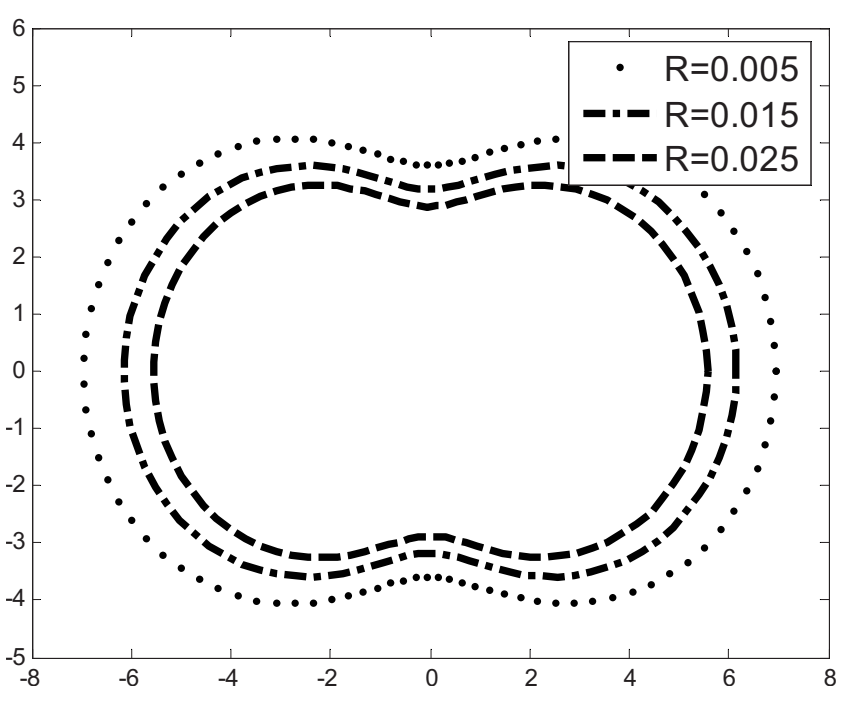

FIG. 3. Optimal electrodes for $\mathrm{ZnO}$ Plates.

poling. The optimal electrodes deviate considerably from common circular or rectangular shapes. They are also significantly different from the nearly elliptical optimal electrodes for quartz plate resonators. ${ }^{7-9}$ Better device performance is expected when optimal electrodes are used.

${ }^{1}$ S. Y. Pao, R. Huang, and C. S. Lam, in Piezoelectricity, Acoustics Waves and Device Applications, edited by J. Wang and W. Q. Chen (World Scientific, Singapore, 2007), p. 152.

${ }^{2}$ J. S. Yang, H. Xue, H. Y. Fang, Y. T. Hu, J. Wang, and L. J. Shen, IEEE Trans. Ultrason. Ferroelectr. Freq. Control 54, 892 (2007).

${ }^{3}$ J. Wang, L. J. Shen, and J. S. Yang, Ultrasonics 48, 150 (2008).

${ }^{4}$ J. S. Yang, Z. G. Chen, and H. P. Hu, Acta Mech. Solida Sinica 20, 266 (2007).

${ }^{5}$ J. S. Yang, Z. G. Chen, and Y. T. Hu, IEEE Trans. Ultrason. Ferroelectr. Freq. Control 55, 841 (2008).

${ }^{6}$ J. S. Yang, H. Xue, and Y. T. Hu, Ferroelectr. Lett. Sect. 34, 108 (2007).

${ }^{7}$ R. D. Mindlin, J. Acoust. Soc. Am. 43, 1329 (1968).

${ }^{8}$ Z. T. Yang and J. S. Yang, IEEE Trans. Ultrason. Ferroelectr. Freq. Control 56, 237 (2009).

${ }^{9}$ Z. T. Yang, J. S. Yang, and Y. T. Hu, Appl. Phys. Lett. 92, 103516 (2008)

${ }^{10}$ R. D. Mindlin, Int. J. Solids Struct. 8, 895 (1972).

${ }^{11}$ J. S. Yang, Analysis of Piezoelectric Devices (World Scientific, Singapore, 2006).

${ }^{12}$ J. L. Bleustein and H. F. Tiersten, J. Acoust. Soc. Am. 43, 1311 (1968).

${ }^{13}$ B. A. Auld, Acoustic Fields and Waves in Solids (Wiley, New York, 1973), Vol. 1. 